\title{
A Receiver Model for Optical Fiber Communication Systems With Arbitrarily Polarized Noise
}

\author{
Ivan T. Lima, Jr., Member, IEEE, Member, OSA, Aurenice O. Lima, Student Member, IEEE, Yu Sun, Hua Jiao, \\ John Zweck, Curtis R. Menyuk, Fellow, IEEE, Fellow, OSA, and Gary M. Carter
}

\begin{abstract}
The authors have derived a receiver model that provides an explicit relationship between the $Q$ factor and the optical signal-to-noise ratio (OSNR) in optical fiber communication systems for arbitrary pulse shapes, realistic receiver filters, and arbitrarily polarized noise. It is shown how the system performance depends on both the degree of polarization of the noise and the angle between the Stokes' vectors of the signal and the noise. The results demonstrate that the relationship between the OSNR and the $Q$ factor is not unique when the noise is partially polarized. This paper defines the enhancement factor and three other parameters that explicitly quantify the relative performance of different modulation formats in a receiver. The theoretical and experimental results show that the performance of the return-to-zero format is less sensitive to variations in the receiver characteristics than is the performance of the nonreturn-to-zero format. Finally, a validation of the formula is presented for computing the $Q$ factor from the OSNR and the Stokes vectors of the signal and the noise by comparison with both experiments and Monte Carlo simulations.
\end{abstract}

Index Terms-Bit-error rate (BER), modulation format, optical communications, optical signal-to-noise ratio (OSNR), polarization, polarization-sensitive devices, $Q$ factor.

\section{INTRODUCTION}

A FUNDAMENTAL problem in the design of optical fiber transmission systems is to achieve a desired bit-error rate (BER), with a given outage probability, after the signal is transmitted through a system. Another widely used performance measure is the $Q$ factor [1], which is a function of the means and the standard deviations of the received electric currents in the marks and in the spaces [1]-[3]. Therefore, the $Q$ factor can be obtained experimentally in the time domain using an oscilloscope. The $Q$ factor can be used to give an approximate value for the BER under the assumption that the electric currents in the marks and in the spaces at the receiver are both Gaussian distributed. Even though the actual distributions of the marks

Manuscript received May 17, 2004; revised September 9, 2004. This work was supported in part by Science Applications International Corporation, the U.S. Department of Energy, and the National Science Foundation.

I. T. Lima, Jr., is with the Department of Electrical and Computer Engineering, North Dakota State University, Fargo, ND 58105-5285 USA (e-mail: Ivan.Lima@ndsu.edu).

A. O. Lima is with the Department of Computer Science and Electrical Engineering, University of Maryland Baltimore County, Baltimore, MD, 21250 USA, and also with the Department of Electrical and Computer Engineering, North Dakota State University, Fargo, ND 58105-5285 USA.

Y. Sun is with Optium Corporation, Chalfont, PA, 18914 USA.

H. Jiao, C. R. Menyuk, and G. M. Carter are with the Department of Computer Science and Electrical Engineering, University of Maryland Baltimore County, Baltimore, MD, 21250 USA.

J. Zweck is with the Department of Mathematics and Statistics, University of Maryland Baltimore County, Baltimore, MD 21250 USA

Digital Object Identifier 10.1109/JLT.2004.839972 and of the spaces are not Gaussian, this approach can provide a good estimate of the BER in some cases [4], [5]. When the BER can be directly measured in the receiver BER $>10^{-13}$, the $Q$ factor can be estimated from the BER [3]. Otherwise, in experiments, the $Q$ factor is usually estimated using the decision-circuit method that was introduced by Bergano et al., [5]. In both these cases, the reported $Q$ factor is really just a restatement of the BER, rather than an independent performance measure. The optical signal-to-noise ratio (OSNR) is another commonly used performance indicator that is even easier to measure than the $Q$ factor [4], [6]. However, the relationship between the OSNR and the $Q$ factor is not straightforward, since the $Q$ factor also depends on the shapes of the optical pulses after transmission, the polarization state of the noise, and on the characteristics of the receiver.

The design and performance evaluation of optical fiber communication systems relies just as much on the accuracy and efficiency of receiver models as it does on accurate and efficient modeling of the transmission line [7]. Accurate receiver modeling is especially important when comparing modulation formats [7], [8]. Marcuse [1] and Humblet and Azizog̃lu [4] derived widely used approximate expressions for the $Q$ factor as a function of the signal-to-noise ratio (SNR) of the electric current and as a function of the OSNR, respectively. In both [1] and [4], the authors assume that the receiver consists of a rectangular optical filter, a square-law photodetector, and an integrate-and-dump electrical filter. They also assume that the optical signals have a perfect extinction ratio and that the optical noise is Gaussian and white prior to the optical filter. Finally, they assume that the signal is polarized and that the optical noise is either unpolarized or is completely polarized and is co-polarized with the signal.

In this paper, Marcuse's and Humblet and Azizog̃lu's results are generalized by deriving a formula for the $Q$ factor in terms of the OSNR for an optical signal in a single polarization state with an arbitrary pulse shape immediately prior to the receiver, with an arbitrary optical extinction ratio in the spaces, for arbitrary optical and electrical filters in the receiver and for arbitrarily polarized noise. To do so, the moments of the electric current in the receiver are calculated using an approach that was introduced earlier by Winzer et al. [7] to calculate the BER.

For systems without polarization effects, the optical noise entering the receiver is unpolarized. This case has been extensively treated in the literature [1], [4], [7], [9], [10]. However, the polarization state of the noise can be significantly affected by the presence of polarization-dependent components, such as components with polarization-dependent loss (PDL) and polarization-dependent gain (PDG) [11]. Recently, there has been a sub- 
stantial amount of work that indicates the importance of these effects [12], [13], and the work reported in this paper was motivated by the experimental work reported in [14].

In this paper, the method in [7] is extended to account for arbitrarily polarized noise. Here, it is demonstrated that, for a fixed OSNR, the $Q$ factor can vary widely depending both on the degree of polarization (DOP) of the noise and on the angle between the Stokes' vectors of the signal and the noise. In this paper, it is assumed that the signal is polarized across the bandwidth of the optical filter. Consequently, the proposed formula for the $Q$ factor is valid only for systems in which the polarization-mode dispersion (PMD) is small enough so that PMD-induced pulse spreading and depolarization of the signal does not have a significant impact on the performance of the system. In such systems, it is still possible for the noise within the bandwidth of the optical filter to become partially polarized due to PDL in the amplifiers. An example of such a system is the prototypical transoceanic undersea system studied in [15], which has low-PMD fiber and a large number of amplifiers, each with a small amount of PDL. In this system, the probability that the degree of polarization of the noise at the receiver exceeds 0.3 is larger than $10^{-3}$. For such a system, a receiver that accounts for partially polarized noise is necessary to accurately calculate the low probability tails of the distribution of the $Q$ factor [16]. A closely related example is the long-haul dispersion-managed soliton system studied in [17] in which the signal remains highly polarized over long distances, since any PMD-induced pulse spreading and depolarization of the signal is counteracted by the strong nonlinearity. Indeed, in [17], our receiver model was used to obtain excellent agreement between simulations and experiments over $18000 \mathrm{~km}$. Such good agreement would not have been possible without a receiver model that accounted for partially polarized noise. Therefore, although there are important situations in which both the signal and the noise may become depolarized, the assumption used in this paper that the signal is polarized is reasonable, since it holds for an important class of experimental systems. The formula for the $Q$ factor presented here can be used in combination with reduced models of the transmission line to quantify how the performance of a system depends on the combined effects of PMD, PDL, PDG and the gain saturation of optical amplifiers [15], [18]. In particular, in [17], the expression for the $Q$ factor that was derived in this paper was used, together with the reduced Stokes' model of the polarization effects [15], to model the performance of a dispersion-managed soliton optical fiber recirculating loop. Excellent agreement was obtained between simulations and experiments at a transmission distance of $18000 \mathrm{~km}$.

In order to correctly account for the pulse shape in the formula for the $Q$ factor, an enhancement factor was defined that explicitly quantifies how efficiently the combination of a pulse shape and a receiver translates the OSNR into the SNR of the electric current in the receiver.

For the results presented in this paper, any intersymbol interference (ISI) in the receiver was accounted for by computing the $Q$ factor using the mark with the smallest voltage and the space with the largest voltage in the noise-free electrically filtered signal [9], [10]. However, in the presence of transmission-induced bit-pattern dependences, one must use the formulas for the mean and the variance of the electric current derived to compute the $Q$ factor more accurately using the procedure introduced in [19]. In other words, using the formula in this paper, one can incorporate partially polarized noise into receiver models that correctly treat bit-pattern dependences in the noise-free signal. Thus, the results presented here are complementary to those of other authors.

The formula for the $Q$ factor is validated by comparison with several experiments and Monte Carlo simulations. Using the authors' own theory and back-to-back experiments, it is shown that the return-to-zero (RZ) format is less sensitive to variations in the receiver characteristics than the nonreturn-to-zero (NRZ) format. In [8], a simplified version of this formula was used to explicitly quantify the advantage in the receiver of using a chirped return-to-zero (CRZ) modulation format rather than an RZ or an NRZ format with the same mean optical power and receiver characteristics. Just as in [1]-[4], the computational cost of computing the $Q$ factor using this formula is orders of magnitude less than the cost of accurately computing the $Q$ factor in the time domain using Monte Carlo simulations. However, this model does not take into account nonlinear signal-noise interactions during transmission that can both amplify and color the noise prior to the receiver in long-haul fiber transmission systems [20], [21].

In Section II, the formula for the $Q$ factor is derived. In that formula, the $Q$ factor is expressed in terms of the SNR of the electric current, and it is extended to express the $Q$ factor in terms of the OSNR and the polarization state of the noise relative to that of the signal. In Sections III and IV, the receiver model discussed in Section II is validated by comparison to Monte Carlo simulations of the receiver and back-to-back experiments, respectively. In both sections, the validation is performed with unpolarized optical noise and with partially polarized noise prior to the receiver. The formula for the $Q$ factor includes parameters, such as the enhancement factor, that only depend on the shape of the noise-free signal and the receiver filters. In the Appendix, tables are provided for these parameters that allow one to easily calculate the $Q$ factor for such systems without having to compute the multiple integrals described in Section II, which are required for the computation of these parameters.

\section{RECEIVER MODEL}

\section{A. Introduction}

In this section, we derive an expression that relates the $Q$ factor to the OSNR, and we introduce the enhancement factor. The enhancement factor quantifies the relative performance of different modulation formats and receivers [15]. We begin by recalling the expressions for the mean and variance of the electrically filtered current in the receiver as in [7], which we generalize to account for arbitrarily polarized optical noise. We emphasize that one cannot simply compute the variance of the electric current due to the beating of the noise with itself by summing the variance of the current that is produced by the noise component that is co-polarized with the signal with the variance of the current produced by the noise component that is orthogonal to the polarization state of the signal. This approach is not 
correct in general, since these two orthogonal components of the noise may be correlated in systems with PDL. Instead, one must decompose the noise into two uncorrelated orthogonal components, neither of which can be assumed to be co-polarized with the signal. However, in Section II-B, we show that this approach can only produce accurate results when the PMD-induced depolarization of the noise within the bandwidth of the optical filter is negligible.

At the receiver, we assume that noise from optical amplifiers is the dominant source of noise, as is the case in an optical communications system with an optically preamplified receiver [22]. Prior to the optical filter in the receiver, we assume that the signal is polarized and that the noise is a Gaussian white noise process that has been generated by optical amplifiers. We let $\mathbf{e}_{s}(t)$ and $\mathbf{e}_{n}(t)$ denote the Jones vectors of the electric-field envelopes of the signal and noise, respectively, prior to the receiver, where $t$ is time. Since most optical transmission systems have polarization-dependent components that can affect the polarization state of the noise, we assume that the optical noise entering the receiver has an arbitrary polarization state.

Our receiver model consists of an optical filter with a complex equivalent baseband transfer function $H_{o}(\omega)$ and corresponding impulse response $h_{o}(t)$, a square-law photodetector, and an electrical filter with a transfer function $H_{e}(\omega)$ and corresponding impulse response $h_{e}(t)$. In our derivation, we have neglected the gain and attenuation in the optical filter and in the electrical circuit of the receiver at the central frequency of the channel, since they do not affect the SNR. Therefore, the absolute value of the transfer functions of the optical and the electrical filters have the value 1 at the central frequency of the channel $\left|H_{o}(0)\right|=\left|H_{e}(0)\right|=1$. The transfer function $H_{e}(\omega)$ can account for the linear effects in other electrical components of the receiver, including the photodetector. Then, the electric current at the receiver is given by

$$
i(t)=R\left|\left[\mathbf{e}_{s}(t)+\mathbf{e}_{n}(t)\right] * h_{o}(t)\right|^{2} * h_{e}(t)
$$

where $R$ is the responsivity of the photodetector, and the convolution of two arbitrary functions $g(t)$ and $h(t)$ is defined by $g(t) * h(t)=\int_{-\infty}^{+\infty} g(\tau) h(t-\tau) \mathrm{d} \tau$

\section{B. Noise Correlation}

We assume that the optical noise prior to the optical filter at the receiver is a Gaussian white noise process that has been generated by optical amplifiers. Therefore, the optical noise is delta-correlated with independent and identically distributed real and imaginary Gaussian probability density functions with zero mean [23]. Hence, the autocorrelation function of the optical noise is given by

$$
\left\langle\mathbf{e}_{n}(t) \cdot \mathbf{e}_{n}^{*}\left(t^{\prime}\right)\right\rangle=N_{\mathrm{ASE}} \delta\left(t-t^{\prime}\right)
$$

where $\mathbf{v} \cdot \mathbf{w}^{*}=v_{1} w_{1}^{*}+v_{2} w_{2}^{*}$ is the standard Hermitian inner product of two Jones vectors $\mathbf{v}=\left[v_{1}, v_{2}\right]^{t}$ and $\mathbf{w}=\left[w_{1}, w_{2}\right]^{t}$, which is independent of the choice of the orthonormal basis of Jones space. The bracket $\langle\cdot\rangle$ indicates an average over all noise realizations, and $N_{\mathrm{ASE}}$ is the total power spectral density of the noise prior to the receiver. The effect of the optical filter on the input light is given by the convolution of the impulse response of the optical filter $h_{o}(t)$ with the Jones vector of the input light. Therefore, the optically filtered noise $\mathbf{e}_{n_{o}}(t)$ can be defined as $\mathbf{e}_{n_{o}}(t)=\mathbf{e}_{n}(t) * h_{o}(t)$. Using (2), the autocorrelation function of the optically filtered noise is given by

$$
\left\langle\mathbf{e}_{n_{o}}(t) \cdot \mathbf{e}_{n_{o}}^{*}\left(t^{\prime}\right)\right\rangle=N_{\mathrm{ASE}} r_{o}\left(t^{\prime}-t\right)
$$

where

$$
r_{o}(\tau)=\int_{-\infty}^{+\infty} h_{o}\left(\tau^{\prime}\right) h_{o}^{*}\left(\tau+\tau^{\prime}\right) \mathrm{d} \tau^{\prime}
$$

is the autocorrelation function of the optical filter. The quantity

$$
r_{o}(0)=\int_{-\infty}^{+\infty}\left|h_{o}\left(\tau^{\prime}\right)\right|^{2} \mathrm{~d} \tau^{\prime}
$$

is the noise equivalent bandwidth of the optical filter [24], which we also denote by $B_{o}$.

To account for the effect of polarization-dependent components in the transmission line, we must first derive expressions for the autocorrelation function of the noise in the two orthogonal polarization state components in which the noise is uncorrelated. To do so, we compute the temporal coherency matrix [24], which is a $2 \times 2$ complex Hermitian matrix that describes both the time correlation and the polarization state of light. Assuming that the optical noise entering the receiver has an arbitrary polarization state, the temporal coherency matrix $J_{n}(\tau)$ of the optically filtered noise is defined by

$$
J_{n}(\tau)=\left[\begin{array}{cc}
\left\langle e_{n_{o, x}}(t) e_{n_{o, x}}^{*}(t+\tau)\right\rangle & \left\langle e_{n_{o, x}}(t) e_{n_{o, y}}^{*}(t+\tau)\right\rangle \\
\left\langle e_{n_{o, y}}(t) e_{n_{o, x}}^{*}(t+\tau)\right\rangle & \left\langle e_{n_{o, y}}(t) e_{n_{o, y}}^{*}(t+\tau)\right\rangle
\end{array}\right]
$$

where $e_{n_{o, x}}(t)$ and $e_{n_{o, y}}(t)$ are the components of the Jones vector of the optically filtered electric field of the noise $\mathbf{e}_{n_{o}}(t)$ in an orthonormal basis $\left\{\hat{\mathbf{a}}_{x}, \hat{\mathbf{a}}_{y}\right\}$ of Jones space, and $\tau$ is a time delay between the field components. We assume that the noise process is wide-sense stationary. Therefore, $J_{n}(\tau)$ does not depend on the time $t$. Assuming that the differential-group delay between two orthogonal components of the complex envelope of the noise field due to PMD in the transmission line is small compared with the width of the impulse response of the optical filter $h_{o}(t)$, the PMD will not cause a significant depolarization of the polarized component of the noise in the bandwidth of the optical filter. As a consequence, all the elements of the temporal coherency matrix $\mathrm{J}_{n}(\tau)$ have approximately the same time-delay dependence $r_{o}(\tau)$ given by (3). In this case, the state of polarization of the filtered noise can be represented by the coherency matrix $\mathrm{J}_{n}=\mathrm{J}_{n}(0)$ of the optically filtered noise defined as [25]

$$
\mathrm{J}_{n}=\left[\begin{array}{ll}
\left\langle e_{n_{o, x}}(t) e_{n_{o, x}}^{*}(t)\right\rangle & \left\langle e_{n_{o, x}}(t) e_{n_{o, y}}^{*}(t)\right\rangle \\
\left\langle e_{n_{o, y}}(t) e_{n_{o, x}}^{*}(t)\right\rangle & \left\langle e_{n_{o, y}}(t) e_{n_{o, y}}^{*}(t)\right\rangle
\end{array}\right]
$$


and the temporal coherency matrix becomes

$$
\mathrm{J}_{n}(\tau)=\frac{r_{o}(\tau)}{r_{o}(0)} \mathrm{J}_{n}
$$

The elements $J_{n_{x x}}=\left\langle e_{n_{o, x}}(t) e_{n_{o}, x}^{*}(t)\right\rangle$ and $J_{n_{y y}}=$ $\left\langle e_{n_{o, y}}(t) e_{n_{o, y}}^{*}(t)\right\rangle$ in (7) are the intensity of the filtered noise in the $\hat{\mathbf{a}}_{x}$ and $\hat{\mathbf{a}}_{y}$ polarizations, respectively, while $J_{n_{x y}}=\left\langle e_{n_{o, x}}(t) e_{n_{o, y}}^{*}(t)\right\rangle$ is a measure of the correlation between the components of the electric field in the $\hat{\mathbf{a}}_{x}$ and $\hat{\mathbf{a}}_{y}$ polarizations [25]. The optical intensity of the filtered noise $I_{\text {tot }}$ is equal to the trace of the matrix $\mathrm{J}_{n}$

$$
\begin{aligned}
I_{\mathrm{tot}} & =\operatorname{TrJ}_{n}=J_{n_{x x}}+J_{n_{y y}} \\
& =\left\langle e_{n_{o, x}}(t) e_{n_{o, x}}^{*}(t)\right\rangle+\left\langle e_{n_{o, y}}(t) e_{n_{o, y}}^{*}(t)\right\rangle=N_{\mathrm{ASE}} B_{o}
\end{aligned}
$$

and the degree of polarization of the filtered noise is given by

$$
\operatorname{DOP}_{n}=\frac{I_{\text {pol }}}{I_{\text {tot }}}=\left(1-\frac{4 \operatorname{Det} J_{n}}{(\operatorname{TrJ})_{n}^{2}}\right)^{1 / 2}
$$

where $I_{\text {pol }}$ is the optical intensity of the polarized part of the filtered noise [25]. Note that the quantities $I_{\text {pol }}, I_{\text {tot }}$, and $\mathrm{DOP}_{n}$ do not depend on the choice of orthonormal basis of Jones space.

The polarization state of the optically filtered noise can also be characterized by the Stokes' parameters $\mathcal{S}_{n}=\left[S_{n_{0}}, S_{n_{1}}, S_{n_{2}}, S_{n_{3}}\right]^{t}$ of the noise, where $S_{n_{0}}$ is the average power of the noise after it is filtered by an optical filter in the receiver. The filtered noise can be decomposed as the sum of a polarized part with Stokes' parameters $\left[\left|\mathbf{S}_{n}\right|, S_{n_{1}}, S_{n_{2}}, S_{n_{3}}\right]^{t}$ and an unpolarized part with Stokes' parameters $\left[S_{n_{0}}-\left|\mathbf{S}_{n}\right|, 0,0,0\right]^{t}$, where $\mathbf{S}_{n}=\left[S_{n_{1}}, S_{n_{2}}, S_{n_{3}}\right]^{t}$ is the Stokes' vector of the filtered noise. The DOP of the noise given by (10) is the power ratio of the polarized part of the noise to the total noise, i.e., $\mathrm{DOP}_{n}=\left|\mathbf{S}_{n}\right| / S_{n_{0}}$. The Stokes' parameters of the optically filtered noise can be expressed in terms of the noise coherency matrix by the formula

$$
\begin{aligned}
S_{n_{0}} & =J_{n_{x x}}+J_{n_{y y}} \\
S_{n_{1}} & =J_{n_{x x}}-J_{n_{y y}} \\
S_{n_{2}} & =J_{n_{x y}}+J_{n_{y x}} \\
S_{n_{3}} & =i\left(J_{n_{y x}}-J_{n_{x y}}\right) .
\end{aligned}
$$

Since the coherency matrix $J_{n}$ is Hermitian, there is an orthonormal basis $\left\{\hat{\mathbf{a}}_{1}, \hat{\mathbf{a}}_{2}\right\}$ of Jones space in which $\mathrm{J}_{n}$ is diagonal with $J_{n_{11}} \geq J_{n_{22}}$ [25]. This basis simply consists of the unit length eigenvectors of $J_{n}$. In the corresponding frame for Stokes' space, the Stokes' vector of the filtered noise is $\mathbf{S}_{n}=\left[S_{n_{0}} \mathrm{DOP}_{n}, 0,0\right]^{t}$. Consequently, in Jones space, the unit vectors $\hat{\mathbf{a}}_{1}$ and $\hat{\mathbf{a}}_{2}$ are, respectively, parallel and perpendicular to the polarized part of the filtered noise. In the basis $\left\{\hat{\mathbf{a}}_{1}, \hat{\mathbf{a}}_{2}\right\}$, the electric field of the filtered noise is given by

$$
\mathbf{e}_{n_{o}}(t)=e_{n_{o, 1}}(t) \hat{\mathbf{a}}_{1}+e_{n_{o, 2}}(t) \hat{\mathbf{a}}_{2}
$$

where the components $e_{n_{o, 1}}(t)$ and $e_{n_{o, 2}}(t)$ of the filtered noise are uncorrelated, since $\mathrm{J}_{n}$ is diagonal. Moreover, in this basis, (10) simplifies to

$$
\operatorname{DOP}_{n}=\left(J_{n_{11}}-J_{n_{22}}\right) /\left(J_{n_{11}}+J_{n_{22}}\right) .
$$

Using (6)-(10) and (13), we find that

$$
\begin{aligned}
& J_{n_{11}}(\tau)=\frac{1}{2}\left(1+\mathrm{DOP}_{n}\right) N_{\mathrm{ASE}} r_{o}(\tau) \\
& J_{n_{22}}(\tau)=\frac{1}{2}\left(1-\mathrm{DOP}_{n}\right) N_{\mathrm{ASE}} r_{o}(\tau)
\end{aligned}
$$

and $J_{n_{12}}(\tau)=0$. Therefore, the autocorrelation functions of the components of the optically filtered noise are

$$
\left\langle e_{n_{o}, 1}(t) e_{n_{o, 1}}^{*}\left(t^{\prime}\right)\right\rangle=\frac{1}{2}\left(1+\mathrm{DOP}_{n}\right) N_{\mathrm{ASE}} r_{o}\left(t^{\prime}-t\right)
$$

and

$$
\left\langle e_{n_{o, 2}}(t) e_{n_{o, 2}}^{*}\left(t^{\prime}\right)\right\rangle=\frac{1}{2}\left(1-\mathrm{DOP}_{n}\right) N_{\mathrm{ASE}} r_{o}\left(t^{\prime}-t\right)
$$

while the cross correlation is

$$
\left\langle e_{n_{o, 1}}(t) e_{n_{o, 2}}^{*}\left(t^{\prime}\right)\right\rangle=0
$$

where $N_{\mathrm{ASE}}$ is the total power spectral density of the noise prior to the receiver that was introduced in (2).

\section{Moments of the Electric Current}

Since we are assuming that the signal is polarized, we can express the Jones vector of the optically filtered signal $\mathbf{e}_{s_{o}}(t)=$ $\mathbf{e}_{s}(t) * h_{o}(t)$ as $\mathbf{e}_{s_{o}}(t)=e_{s_{o}}(t) \hat{\mathbf{e}}_{s_{o}}$, where $e_{s_{o}}(t)$ is a scalar field and $\hat{\mathbf{e}}_{s_{o}}$ is a unit Jones vector. The expression for $\mathbf{e}_{s_{o}}(t)$ in the basis $\left\{\hat{\mathbf{a}}_{1}, \hat{\mathbf{a}}_{2}\right\}$ is

$$
\mathbf{e}_{s_{o}}(t)=e_{s_{o}}(t)\left(\hat{\mathbf{e}}_{s_{o}} \cdot \hat{\mathbf{a}}_{1}^{*}\right) \hat{\mathbf{a}}_{1}+e_{s_{o}}(t)\left(\hat{\mathbf{e}}_{s_{o}} \cdot \hat{\mathbf{a}}_{2}^{*}\right) \hat{\mathbf{a}}_{2} \cdot
$$

The term $e_{s_{o}}(t)\left(\hat{\mathbf{e}}_{s_{o}} \cdot \hat{\mathbf{a}}_{1}^{*}\right) \hat{\mathbf{a}}_{1}$ is the component of the Jones vector of the filtered signal that is in the same polarization state as the polarized part of the noise, while $e_{s_{o}}(t)\left(\hat{\mathbf{e}}_{s_{o}} \cdot \hat{\mathbf{a}}_{2}^{*}\right) \hat{\mathbf{a}}_{2}$ is the component of the Jones vector of filtered signal that is orthogonal to the polarized part of the noise.

We now substitute the the expressions for $\mathbf{e}_{n_{o}}(t)$ and $\mathbf{e}_{s_{o}}(t)$ in (12) and (18) into (1) to obtain

$$
\begin{aligned}
i(t)=R\left\{\left|\left(\hat{\mathbf{e}}_{s_{o}} \cdot \hat{\mathbf{a}}_{1}^{*}\right) e_{s_{o}}(t)+e_{n_{o}, 1}(t)\right|^{2}\right. \\
\left.\quad+\left|\left(\hat{\mathbf{e}}_{s_{o}} \cdot \hat{\mathbf{a}}_{2}^{*}\right) e_{s_{o}}(t)+e_{n_{o}, 2}(t)\right|^{2}\right\} * h_{e}(t) .
\end{aligned}
$$

In order to compute the mean $\left\langle i_{n}\right\rangle(t)$ and the variance $\sigma_{i}^{2}(t)$ of the current at any time $t$, we use the statistical properties of the optically filtered noise that we described earlier in this section.

Combining (15)-(17) with (19), we find that

$$
\langle i\rangle(t)=i_{s}(t)+\left\langle i_{n}\right\rangle(t)
$$

where $\langle\cdot\rangle(t)$ is the average over the statistical realizations of the noise at time $t$, and

$$
i_{s}(t)=R\left|\mathbf{e}_{s_{o}}(t)\right|^{2} * h_{e}(t)
$$


is the electric current due to the signal. Furthermore,

$$
\left\langle i_{n}\right\rangle(t)=\left\langle i_{n}\right\rangle=R N_{\mathrm{ASE}} B_{o}
$$

is the mean current due to noise, which is time independent, since the optical noise is a wide-sense stationary random process. The parameter $B_{o}=r_{o}(0)=\int_{-\infty}^{+\infty}\left|h_{o}(\tau)\right|^{2} \mathrm{~d} \tau$ is the noise-equivalent bandwidth [24] of the optical filter. If, for example, the noise has a power spectral density of $1 \mathrm{~W} / \mathrm{Hz}$, then after passing an optical filter with noise-equivalent bandwidth $B_{o}$ (in hertz) the optical power is exactly equal to $B_{o}$ (in watts), regardless of the shape of the optical filter. As a consequence, the noise-equivalent bandwidth is a measure of the optical bandwidth that is more useful for the study of optical noise statistics in this context than other more traditional bandwidth measures, such as the full-width at half-maximum (FWHM) and the root-mean-square width.

Following a similar procedure used in the derivation of (22), we find that the variance of the current at any time $t$ has the form

$$
\sigma_{i}^{2}(t)=\left\langle i^{2}\right\rangle(t)-\langle i\rangle^{2}(t)=\sigma_{\mathrm{ASE}-\mathrm{ASE}}^{2}+\sigma_{\mathrm{S}-\mathrm{ASE}}^{2}(t) .
$$

The first term on the right side of (23) is the variance of the current due to the noise beating with itself in the receiver-the noise-noise beating. The variance of the current due to the noise-noise beating is given by

$$
\sigma_{\mathrm{ASE}-\mathrm{ASE}}^{2}=\frac{1}{2} R^{2} N_{\mathrm{ASE}}^{2} \frac{I_{\mathrm{ASE}-\mathrm{ASE}}}{\Gamma_{\mathrm{ASE}-\mathrm{ASE}}}
$$

where

$$
\Gamma_{\mathrm{ASE}-\mathrm{ASE}}=\frac{1}{1+\mathrm{DOP}_{n}^{2}}
$$

and

$$
I_{\mathrm{ASE}-\mathrm{ASE}}=\int_{-\infty}^{+\infty}\left|r_{o}(\tau)\right|^{2} r_{e}(\tau) \mathrm{d} \tau \text {. }
$$

Here, the expression

$$
r_{e}(\tau)=\int_{-\infty}^{+\infty} h_{e}\left(\tau^{\prime}\right) h_{e}\left(\tau+\tau^{\prime}\right) \mathrm{d} \tau^{\prime}
$$

is the autocorrelation function of the electrical filter. The noise-noise beating factor $\Gamma_{\mathrm{ASE}-\mathrm{ASE}}$ is the ratio between the variance of the current due to noise-noise beating in the case that the noise is unpolarized to the actual variance of the current due to noise-noise beating. The second term on the right-hand side of (23) is the variance of the current due to the beating between the signal and the noise in the receiver-the signal-noise beating. The variance of the current due to the signal-noise beating is given by

$$
\begin{aligned}
& \sigma_{\mathrm{S}-\mathrm{ASE}}^{2}(t)=\frac{1}{2} R^{2} N_{\mathrm{ASE}} {\left[\left(1+\mathrm{DOP}_{n}\right)\left|\hat{\mathbf{e}}_{s_{o}} \cdot \hat{\mathbf{a}}_{1}^{*}\right|^{2}\right.} \\
&\left.+\left(1-\mathrm{DOP}_{n}\right)\left|\hat{\mathbf{e}}_{s_{o}} \cdot \hat{\mathbf{a}}_{2}^{*}\right|^{2}\right] I_{\mathrm{S}-\mathrm{ASE}}(t)
\end{aligned}
$$

where

$$
\begin{aligned}
I_{\mathrm{S}-\mathrm{ASE}}(t)= & 2 \int_{-\infty}^{+\infty} e_{s_{o}}(\tau) h_{e}(t-\tau) \\
& \times \int_{-\infty}^{+\infty} e_{s_{o}}^{*}\left(\tau^{\prime}\right) h_{e}\left(t-\tau^{\prime}\right) r_{o}\left(\tau-\tau^{\prime}\right) \mathrm{d} \tau^{\prime} \mathrm{d} \tau .
\end{aligned}
$$

The integral expressions in (26) and (29) may be derived following a procedure similar to the one in [7]. The terms $\left|\hat{\mathbf{e}}_{s_{o}} \cdot \hat{\mathbf{a}}_{1}^{*}\right|^{2}$ and $\left|\hat{\mathbf{e}}_{s_{o}} \cdot \hat{\mathbf{a}}_{2}^{*}\right|^{2}$ are the relative intensities of the components of the field $e_{s_{o}}(t) \hat{\mathbf{e}}_{s_{o}}$ that are, respectively, parallel and perpendicular in Jones space to the polarized part of the optically filtered noise. These terms can be represented in Stokes' space as

$$
\left|\hat{\mathbf{e}}_{s_{o}} \cdot \hat{\mathbf{a}}_{1}^{*}\right|^{2}=\frac{1}{2}\left(1+\mathbf{s}_{s} \cdot \mathbf{s}_{n}^{(p)}\right)
$$

and

$$
\left|\hat{\mathbf{e}}_{s_{o}} \cdot \hat{\mathbf{a}}_{2}^{*}\right|^{2}=\frac{1}{2}\left(1-\mathbf{s}_{s} \cdot \mathbf{s}_{n}^{(p)}\right)
$$

where $\mathbf{s}_{s}$ and $\mathbf{s}_{n}^{(p)}=\mathbf{S}_{n} /\left|\mathbf{S}_{n}\right|$ are the unit Stokes' vectors of the signal and the polarized part of the filtered noise, respectively. The dot products on the right-hand sides of (30) and (31) are the standard dot product between two real valued vectors with three dimensions, which can also be mathematically represented by the definition given after (2). Substituting (30) and (31) into (28), we find that

$$
\sigma_{\mathrm{S}-\mathrm{ASE}}^{2}(t)=R^{2} N_{\mathrm{ASE}} \Gamma_{\mathrm{S}-\mathrm{ASE}} I_{\mathrm{S}-\mathrm{ASE}}(t)
$$

where

$$
\Gamma_{\mathrm{S}-\mathrm{ASE}}=\frac{1}{2}\left[1+\mathrm{DOP}_{n}\left(\mathbf{s}_{s} \cdot \mathbf{s}_{n}^{(p)}\right)\right]
$$

is the signal-noise beating factor, which is the fraction of the noise that beats with the signal.

In the case with the signal, (22), (24), and (32) agree with the corresponding expressions in [7].

\section{The $\mathrm{Q}$ Factor and the Enhancement Factor}

We now use the expressions for the mean and the variance of the current that we derived in Section II-C to derive a general expression for the $Q$ factor as a function of the OSNR. We follow a procedure that is similar to the one described in [1], but we use the exact mean and the exact variance of the electric current. We start with the standard time-domain definition of the $Q$ factor, as follows:

$$
Q=\frac{\left\langle i_{1}\right\rangle-\left\langle i_{0}\right\rangle}{\sigma_{1}+\sigma_{0}}
$$

as in [1], which is related to the BER by $\mathrm{BER} \simeq$ $\operatorname{erfc}(Q / \sqrt{2}) / 2 \simeq \exp \left(-Q^{2} / 2\right) /(\sqrt{2 \pi} Q)$, provided that the electric currents in the marks and in the spaces are Gaussian distributed. 
For the results presented in this paper, we compute the $Q$ factor using the smallest mark and largest space in the noise-free signal, since these two bits are the main sources of errors in the receiver [9], [10]. Rebola and Cartaxo showed that this procedure produces accurate $Q$-factor estimates in systems that do not have significant ISI [10]. Substituting (20) and (23) into (34), we now obtain (35), shown at the bottom of the page, where $t_{1}$ and $t_{0}$ are the sampling times of the lowest mark and the highest space, respectively. To define the sampling time for marks and spaces, we recover the clock using algorithms based on those described by Trischitta and Varma [26]. In the presence of pattern dependences and ISI, one can use the expressions for the moments of the electric current in (22), (24), and (32) to compute a more accurate expression for the $Q$ factor using a procedure that was introduced by Anderson and Lyle [19], which corresponds to the true $Q$ factor in [27].

By rearranging (35), the $Q$ factor can be expressed in terms of the SNR of the electric current of a mark

$$
\mathrm{SNR}_{1}=\frac{i_{s}\left(t_{1}\right)}{\left\langle i_{n}\right\rangle}
$$

and the extinction ratio of the electric current in the receiver

$$
\alpha_{e}=\frac{i_{s}\left(t_{0}\right)}{i_{s}\left(t_{1}\right)}
$$

as

$$
Q=\frac{\left(1-\alpha_{e}\right) \mathrm{SNR}_{1} M^{1 / 2}}{\left(K_{1} \mathrm{SNR}_{1}+1\right)^{1 / 2}+\left(K_{0} \alpha_{e} \mathrm{SNR}_{1}+1\right)^{1 / 2}}
$$

where

and

$$
K_{0}=\frac{\left\langle i_{n}\right\rangle \sigma_{\mathrm{S}-\mathrm{ASE}}^{2}\left(t_{0}\right)}{i_{s}\left(t_{0}\right) \sigma_{\mathrm{ASE}-\mathrm{ASE}}^{2}}
$$

$$
K_{1}=\frac{\left\langle i_{n}\right\rangle \sigma_{\mathrm{S}-\mathrm{ASE}}^{2}\left(t_{1}\right)}{i_{s}\left(t_{1}\right) \sigma_{\mathrm{ASE}-\mathrm{ASE}}^{2}}
$$

We use the definition of the electrical SNR given in (36) since, as we will see in (49) hereafter, it is closely related to the OSNR. We call $K_{0}$ and $K_{1}$ the signal-noise beating parameters for the spaces and marks, respectively, because they are directly proportional to the ratio between the variance of the current due to the signal-noise beating and the variance of the current due to the noise-noise beating. The parameter

$$
M=\frac{\left\langle i_{n}\right\rangle^{2}}{\sigma_{\mathrm{ASE}-\mathrm{ASE}}^{2}}
$$

is the effective number of noise modes. We use this terminology because $M$ is a generalization of the parameter indicating the number of noise modes that was introduced by Marcuse [1]. In that work, Marcuse found that only a finite number of noise Fourier components-noise modes-contribute to the electric current due to noise in a receiver with a rectangular optical filter and an integrate-and-dump electrical filter.

The parameters $K_{0}, K_{1}$, and $M$ are dimensionless and do not depend on the average power of the signal or on the average power of the noise. To separate out the dependence of these three parameters on the polarization state of the noise, we use (24) and (32) to express $K_{0}$ and $K_{1}$ as

$$
K_{0}=2 \Gamma_{\mathrm{S}-\mathrm{ASE}} \Gamma_{\mathrm{ASE}-\mathrm{ASE}} \kappa_{0}
$$

and

$$
K_{1}=2 \Gamma_{\mathrm{S}-\mathrm{ASE}} \Gamma_{\mathrm{ASE}-\mathrm{ASE}} \kappa_{1}
$$

where

and

$$
\kappa_{0}=\frac{R B_{o} I_{\mathrm{S}-\mathrm{ASE}}\left(t_{0}\right)}{i_{s}\left(t_{0}\right) I_{\mathrm{ASE}-\mathrm{ASE}}}
$$

$$
\kappa_{1}=\frac{R B_{o} I_{\mathrm{S}-\mathrm{ASE}}\left(t_{1}\right)}{i_{s}\left(t_{1}\right) I_{\mathrm{ASE}-\mathrm{ASE}}} .
$$

If the noise is either unpolarized or completely co-polarized with the signal, then $K_{0}$ and $K_{1}$ are equal to $\kappa_{0}$ and $\kappa_{1}$, respectively, since then $2 \Gamma_{\mathrm{S}-\mathrm{ASE}} \Gamma_{\mathrm{ASE}-\mathrm{ASE}}=1$. Similarly, we obtain $M=\Gamma_{\mathrm{ASE}-\mathrm{ASE}} \mu$, where

$$
\mu=\frac{2 B_{o}^{2}}{I_{\mathrm{ASE}-\mathrm{ASE}}}
$$

is equal to $M$ in the case where the noise is unpolarized. Then, the $Q$ factor can be expressed as (47), shown at the bottom of the

$$
Q=\frac{\left[i_{s}\left(t_{1}\right)+\left\langle i_{n}\right\rangle\right]-\left[i_{s}\left(t_{0}\right)+\left\langle i_{n}\right\rangle\right]}{\left(\sigma_{\mathrm{S}-\mathrm{ASE}}^{2}\left(t_{1}\right)+\sigma_{\mathrm{ASE}-\mathrm{ASE}}^{2}\right)^{1 / 2}+\left(\sigma_{\mathrm{S}-\mathrm{ASE}}^{2}\left(t_{0}\right)+\sigma_{\mathrm{ASE}-\mathrm{ASE}}^{2}\right)^{1 / 2}}
$$

$$
Q=\frac{\left(1-\alpha_{e}\right) \mathrm{SNR}_{1}\left(\Gamma_{\mathrm{ASE}-\mathrm{ASE}} \mu\right)^{1 / 2}}{\left(2 \Gamma_{\mathrm{S}-\mathrm{ASE}} \Gamma_{\mathrm{ASE}-\mathrm{ASE}} \kappa_{1} \mathrm{SNR}_{1}+1\right)^{1 / 2}+\left(2 \Gamma_{\mathrm{S}-\mathrm{ASE}} \Gamma_{\mathrm{ASE}-\mathrm{ASE}} \kappa_{0} \alpha_{e} \mathrm{SNR}_{1}+1\right)^{1 / 2}}
$$


page. In this formula, the dependence on the polarization state of the noise is accounted for by the noise-noise beating factor $\Gamma_{\text {ASE-ASE }}$ defined in (25) and the signal-noise beating factor $\Gamma_{\mathrm{S}-\mathrm{ASE}}$ given in (33). These factors have values in the range $1 / 2 \leq \Gamma_{\mathrm{ASE}-\mathrm{ASE}} \leq 1$ and $0 \leq \Gamma_{\mathrm{S}-\mathrm{ASE}} \leq 1$. If the noise is unpolarized, $\Gamma_{\mathrm{ASE}-\mathrm{ASE}}=1$ and $\Gamma_{\mathrm{S}-\mathrm{ASE}}=1 / 2$. If the noise is completely co-polarized with the signal, $\Gamma_{\mathrm{ASE}-\mathrm{ASE}}=1 / 2$ and $\Gamma_{\mathrm{S}-\mathrm{ASE}}=1$.

The expression for the $Q$ factor in (47) separates the effects of the polarization state of the noise and of the signal from the pulse shape and the receiver characteristics. However, the $Q$ factor is given as a function of the $\mathrm{SNR}$ of the marks $\mathrm{SNR}_{1}$ in the receiver, which also depends on the pulse shape and on the characteristics of the receiver. Thus, it is appropriate to express the $Q$ factor in terms of a quantity that does not depend on the pulse shape or on the receiver, such as the OSNR. We define the OSNR by

$$
\text { OSNR }=\frac{\left\langle\left|e_{s}(t)\right|^{2}\right\rangle_{t}}{N_{\mathrm{ASE}} B_{\mathrm{OSA}}}
$$

where $\left\langle\left|e_{s}(t)\right|^{2}\right\rangle_{t}$ is the time-averaged noiseless optical power per channel prior to the optical filter, and $B_{\mathrm{OSA}}$ is the noise equivalent bandwidth of an optical spectrum analyzer (OSA) that is used to measure the optical power of the noise. This definition of OSNR is consistent with the definition in [6], [28] and agrees with the OSNR value that can be obtained directly from an OSA whose resolution bandwidth is large compared with the bandwidth of the signal.

In order to express the $Q$ factor as a function of the OSNR, we define the enhancement factor $\xi$ as the ratio between the SNR of the electric current of the marks $\mathrm{SNR}_{1}$ and the OSNR at the receiver. The enhancement factor can be expressed as

$$
\xi=\frac{\mathrm{SNR}_{1}}{\mathrm{OSNR}}=\frac{i_{s}\left(t_{1}\right)}{\left\langle i_{n}\right\rangle} \frac{N_{\mathrm{ASE}} B_{\mathrm{OSA}}}{\left\langle\left|e_{s}(t)\right|^{2}\right\rangle_{t}}=\xi^{\prime} \frac{B_{\mathrm{OSA}}}{B_{o}}
$$

where $\xi^{\prime}=i_{s}\left(t_{1}\right) /\left[R\left\langle\left|e_{\mathrm{in}}(t)\right|^{2}\right\rangle_{t}\right]$ is the normalized enhancement factor, which is equal to $\xi$ when $B_{\mathrm{OSA}}=B_{o}$. The enhancement factor quantifies how efficiently the combination of the pulse shape and receiver translates the OSNR into the SNR of the electric current of the marks in the receiver.

Substituting (49) into (47), we finally obtain an exact expression that relates the $Q$ factor directly to the OSNR and to the polarization states of the optical noise and of the signal prior to the receiver, as shown in (50) at the bottom of the page. The optical pulse shape prior to the receiver and the shapes and bandwidths of the optical and electrical filters in the receiver are taken into account in the determination of the values for $\kappa_{0}, \kappa_{1}$ and $\mu$, as given in (44)-(46).

\section{E. Comparison With Previous Formulas for the Q Factor}

In an optical fiber system with the NRZ modulation format that consists of perfectly rectangular pulses with perfect optical extinction ratio $\left(\alpha_{o}=0\right)$, unpolarized optical noise, and a receiver that consists of a rectangular optical filter and an integrate-and-dump electrical filter, the formula (47) for $Q$ becomes

$$
Q=\frac{\mathrm{SNR}_{1}}{\left(\kappa_{1} \mathrm{SNR}_{1}+1\right)^{1 / 2}+1} \sqrt{\mu}
$$

where $\kappa_{1}=2$, which is the same as the formula for $Q$ in [1]. Using (50) and (51), we can express the $Q$ factor in terms of the OSNR as

$$
Q=\frac{\xi \mathrm{OSNR}}{\left(\kappa_{1} \xi \mathrm{OSNR}+1\right)^{1 / 2}+1} \sqrt{\mu}
$$

where $\kappa_{1}=2$ and $\xi=2$, which is the same as the formula for $Q$ in [6] for the same system that we considered in (51). In (52), $\xi=2$ because the average optical power in this system is equal to half of the average optical power in the marks.

\section{F. Numerical Efficiency}

To efficiently compute the parameters $\kappa_{0}, \kappa_{1}$, and $\mu$, we can use Fourier transforms to numerically compute the multiple integrals in (26) and (29). From the convolution theorem, we obtain

$$
I_{\mathrm{ASE}-\mathrm{ASE}}=\int_{-\infty}^{+\infty}\left|\mathcal{F}_{\tau}^{-1}\left\{\left|\tilde{H}_{o}\right|^{2}\right\}\right|^{2} \mathcal{F}_{\tau}^{-1}\left\{\left|H_{e}\right|^{2}\right\} \mathrm{d} \tau
$$

where $\tilde{H}_{o}(\omega)=H_{o}(-\omega)$, and $\mathcal{F}_{\tau}[\cdot]$ and $\mathcal{F}_{\tau}^{-1}\{\cdot\}$ denote the forward and inverse Fourier transform with respect to $\tau$, while

$$
\begin{aligned}
I_{\mathrm{S}-\mathrm{ASE}}(t)=2 \mathcal{F}_{t}^{-1}\{ & H_{e} \mathcal{F}_{\tau}\left[e_{s_{o}}(\tau)\right. \\
& \left.\left.\times \mathcal{F}_{\tau}^{-1}\left\{\left|\tilde{H}_{o}\right|^{2} \mathcal{F}_{\tau^{\prime}}\left[e_{s_{o}}^{*}\left(\tau^{\prime}\right) h_{e}\left(t-\tau^{\prime}\right)\right]\right\}\right]\right\}
\end{aligned}
$$

where $h_{e}(t)=\mathcal{F}_{t}^{-1}\left\{H_{e}\right\}$.

To correctly interpret (54), we first observe that the convolution operation with respect to $\tau$

$$
\left[g(\tau, t) *_{\tau} h(\tau)\right](t)=\int_{-\infty}^{+\infty} g(\tau, t) h(t-\tau) \mathrm{d} \tau
$$

where we regard $t$ as a parameter in the function $g(\tau, t)$, can be expressed as

$$
\left[g(\tau, t) *_{\tau} h(\tau)\right](t)=\mathcal{F}_{\tau}^{-1}\left[\left(\mathcal{F}_{\tau} g\right)(\omega, t)\left(\mathcal{F}_{\tau} h\right)(\omega)\right] .
$$

$$
Q=\frac{\left(1-\alpha_{e}\right) \xi \mathrm{OSNR}\left(\Gamma_{\mathrm{ASE}-\mathrm{ASE}} \mu\right)^{1 / 2}}{\left(2 \Gamma_{\mathrm{S}-\mathrm{ASE}} \Gamma_{\mathrm{ASE}-\mathrm{ASE}} \kappa_{1} \xi \mathrm{OSNR}+1\right)^{1 / 2}+\left(2 \Gamma_{\mathrm{S}-\mathrm{ASE}} \Gamma_{\mathrm{ASE}-\mathrm{ASE}} \kappa_{0} \alpha_{e} \xi \mathrm{OSNR}+1\right)^{1 / 2}}
$$




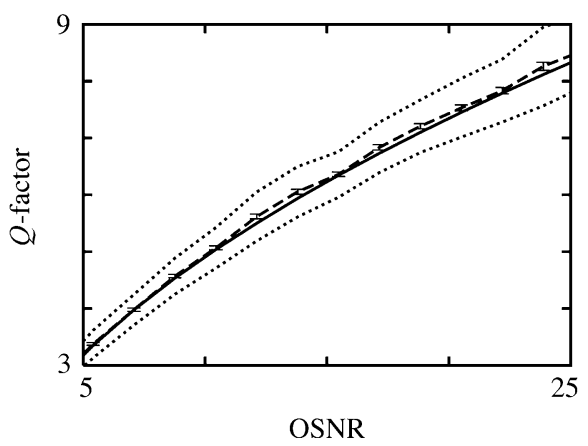

Fig. 1. Comparison of the formula (50) for the $Q$ factor as a function of the OSNR with the time-domain Monte Carlo method of computing the $Q$ factor for the $\mathrm{RZ}$ raised-cosine format. The noise-equivalent bandwidth of the OSA was $25 \mathrm{GHz}$. For the Monte Carlo simulations, the statistics of the $Q$ factor were obtained using $100 Q$ samples each with $128 \mathrm{~b}$. The solid line shows the result using (50). The dashed line and the two dotted lines show the mean $Q$ factor for all $100 Q$ samples and the confidence interval for a single $Q$ sample, defined by the mean $Q$ factor plus and minus one standard deviation computed using the time-domain Monte Carlo method. The error bars show the confidence interval for the mean $Q$ factor for all $100 Q$ samples.

Therefore, for each fixed value of $t$, a discrete approximation to (55) is given by the inverse discrete Fourier transform (DFT) of the product of the DFTs of the vectors $g\left(\tau_{i}, t\right)$ and $h\left(\tau_{i}\right)$. Then, to interpret (54), we observe that

$$
\begin{aligned}
I_{\mathrm{S}-\mathrm{ASE}}(t)=2\left\{h_{e}(\tau) *_{\tau}\right. & {\left[e _ { s _ { o } } ( \tau ) \left(\left\{e_{s_{o}}^{*}\left(\tau^{\prime}\right)\right.\right.\right.} \\
& \left.\left.\left.\left.\times h_{e}\left(t-\tau^{\prime}\right)\right\} *_{\tau^{\prime}} r_{o}\left(\tau^{\prime}\right)\right)(\tau)\right]\right\}(t) .
\end{aligned}
$$

\section{RECEIVER MODEL VALIDATION WITH Monte CARlo Simulations}

We now present a validation of (50) for computing the $Q$ factor from the OSNR with a known pulse shape at the receiver by comparison with two sets of Monte Carlo simulations in which the $Q$ factor is computed using the standard time-domain formula $Q=\left(\left\langle i_{1}\right\rangle-\left\langle i_{0}\right\rangle\right) /\left(\sigma_{1}+\sigma_{0}\right)$. For the first set of simulations, we used a back-to-back $10-\mathrm{Gb} / \mathrm{s}$ optical system with unpolarized optical noise that was added prior to the receiver using a Gaussian noise source that has a constant spectral density within the spectrum of the optical filter. For the second set of simulations, we used another back-to-back $10-\mathrm{Gb} / \mathrm{s}$ system with partially polarized optical noise, which was obtained by transmitting unpolarized noise through a PDL element. Since our study is focused on the combined effect that the pulse shape and the receiver have on the system performance, we did not include transmission effects here, such as those due to nonlinearity and dispersion.

In Fig. 1, we show the $Q$ factor versus the OSNR for an RZ raised-cosine format with a $50 \%$ duty cycle and an optical extinction ratio of $18 \mathrm{~dB}$. The electric field of an $\mathrm{RZ}$ raised-cosine pulse is given by $e_{s}(t)=\left[P_{0} \cos ^{2}(\pi t / T)\right]^{1 / 2}$, where $P_{0}$ is the peak power and $T$ is the bit period. The receiver consisted of a Gaussian-shaped optical filter with a FWHM of $124 \mathrm{GHz}$ and a fifth-order, low-pass electrical Bessel filter with a 3-dB bandwidth of $8.5 \mathrm{GHz}$. The noise-equivalent bandwidth [24]

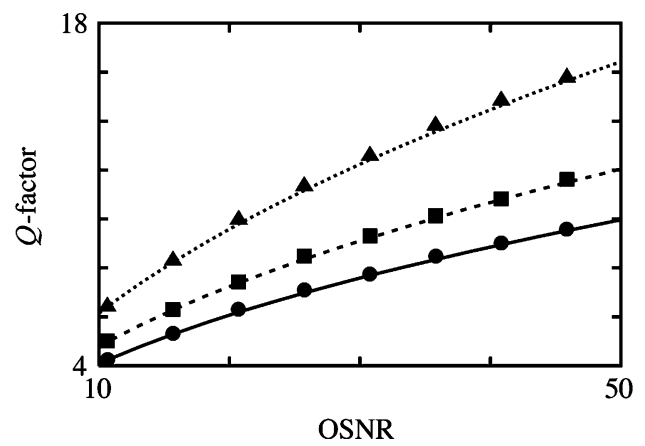

Fig. 2. Comparison of the formula (50) with the time-domain Monte Carlo method for computing the $Q$ factor as a function of the OSNR for the RZ raised-cosine format for different noise polarization states with $\mathrm{DOP}_{n}=0.5$. These results are for a horizontally polarized optical signal. The noise-equivalent bandwidth of the OSA was $25 \mathrm{GHz}$. The curves show the results obtained using (50), and the symbols show the results obtained using Monte Carlo simulations. The solid curve and the circles show the results when the polarized part of the noise is co-polarized with the signal. Similarly, the dashed curve and the squares as well as the dotted curve and the triangles show the results when the polarized part of the noise is in the left circular and orthogonal linearly polarized states, respectively.

of the OSA was equal to $25 \mathrm{GHz}$. The parameters in (50) for this system are $\xi=0.6, \alpha_{e}=-18 \mathrm{~dB}, \kappa_{0}=\kappa_{1}=3, \mu=$ 21.23, $\Gamma_{\mathrm{ASE}-\mathrm{ASE}}=1$, and $\Gamma_{\mathrm{S}-\mathrm{ASE}}=1 / 2$.

In Fig. 1, we show the results using (50) with a solid line. We obtained these results using only a single mark and a single space of the transmitted bit string. We show the results for the time-domain Monte Carlo method with a dashed line. We obtained these results by averaging over 100 samples of the $Q$ factor, where for each sample the means and standard deviations of the marks and spaces were estimated using $128 \mathrm{~b}$. The agreement between the two methods is excellent, except for the statistical error in the computation of the $Q$ factor using the time-domain Monte Carlo method. The numerical estimator of the standard deviation of the current due to noise using the time-domain Monte Carlo method is a biased estimator [29], which contributes to the small systematic error in the estimation of the $Q$ factor using the time-domain Monte Carlo method. The region between the dotted lines in Fig. 1 is the confidence interval for the computation of the $Q$ factor using the time-domain Monte Carlo method for a string with $128 \mathrm{~b}$. The confidence interval, defined by the mean $Q$ factor plus and minus one standard deviation of the $Q$ factor, gives an estimate of the error in the computation of the $Q$ factor using the time-domain Monte Carlo method with a single string of bits. Since we used 100 strings to obtain an estimate of the $Q$ factor using the time-domain Monte Carlo method in Fig. 1, its confidence interval is ten times smaller [29] than the $Q$ factor computed from a single bit string. In this figure, we show the confidence interval of the mean $Q$ factor with error bars. In Fig. 1, the time-domain Monte Carlo method obtained with a single bit string has a relative statistical uncertainty larger than $15 \%$ with a single string when $Q=6$.

For the second set of simulations, we used partially polarized noise. In Fig. 2, we plot the $Q$ factor versus the OSNR for a linearly polarized RZ raised-cosine signal with an optical extinction ratio of $18 \mathrm{~dB}$. We used a $10-\mathrm{Gb} / \mathrm{s}$ back-to-back system and added partially polarized noise with $\mathrm{DOP}_{n}=0.5$ prior to the 


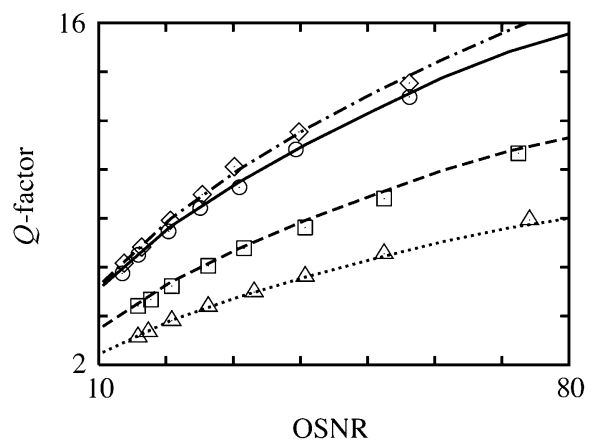

Fig. 3. Comparison of the $Q$ factor as a function of the OSNR obtained using (50) with experimental results for different modulation formats and receivers. The noise-equivalent bandwidth of the OSA was $25 \mathrm{GHz}$. The curves show the results obtained using (50), and the experimental results are shown using symbols. The dotted-dashed curve and the diamonds show the results for the RZ format with an electrical filter with a 3-dB bandwidth of $7 \mathrm{GHz}$. The solid curve and circles show the results for the RZ format without the electrical filter. The dashed curve and the squares show the results for the NRZ format with an electrical filter with a 3-dB bandwidth of $7 \mathrm{GHz}$. The dotted curve and the triangles show the results for the NRZ format without the electrical filter.

receiver. The receiver and the optical spectrum analyzer bandwidth were the same as that used for Fig. 1. In Fig. 2, the curves show the results obtained using (50) and the symbols show the results obtained using Monte Carlo simulations. The solid curve and circles show the results when the polarized part of the noise is co-polarized with the signal. Similarly, the dashed curve and the squares, and the dotted curve and the triangles show the results when the polarized part of the noise is in the left circular and orthogonal linearly polarized states, respectively. The agreement between (50) and Monte Carlo simulations is excellent. When $\mathrm{DOP}_{n}=0.5$, the $Q$ factor varies by about $60 \%$ as we vary the polarization state of the noise. This variation occurs because the signal-noise beating factor $\Gamma_{\mathrm{S}-\mathrm{ASE}}$ in (33) depends on the angle between the Stokes' vectors of the signal and the polarized part of the noise. The parameters in (50) for this system are the same ones in Fig. 1, except that $\Gamma_{\mathrm{ASE}-\mathrm{ASE}}=0.8$ and $\Gamma_{\mathrm{S}-\mathrm{ASE}}=1$ for the solid line, $\Gamma_{\mathrm{S}-\mathrm{ASE}}=0.5$ for the dashed line, and $\Gamma_{\mathrm{S}-\mathrm{ASE}}=0.25$ for the dotted line.

\section{RECEIVER MODEL VALIDATION WITH EXPERIMENTS}

We now present a validation of (50) by comparison with two sets of back-to-back 10-Gb/s experiments. In the first set of experiments, the noise is unpolarized $\left(\mathrm{DOP}_{n} \simeq 0\right)$. In the second set of experiments, for which the noise is partially polarized (DOP $\left.{ }_{n} \in[0,1]\right)$, the $Q$ factor depends strongly on the polarization state of the noise when the noise is partially polarized. In both cases, the experiments agree well with our formula.

In Fig. 3, we plot the $Q$ factor versus the OSNR obtained using both simulations and experiments for RZ and NRZ signals with unpolarized optical noise $\left(\mathrm{DOP}_{n}<0.05\right)$ that is generated by an erbium-doped fiber amplifier (EDFA) without input power [30]. In the transmitter, we generated a $10-\mathrm{Gb} / \mathrm{s}$ pulse train using an electroabsorption modulator (EAM). The data was encoded on the pulse train using an electrooptic modulator (EOM). For the NRZ signal, the EAM was bypassed. To avoid pattern dependences in the EOM modulator that we used to encode the data, we used the fixed pattern 01010101 . Therefore, these results
TABLE I

PARAMETERS OF THE MOdULATION Formats USED IN FIG. 3 With AND WiTHOUT ELECTRICAL FILTER (EF)

\begin{tabular}{l||r|r|r|r|r|r}
\hline Format & $\alpha_{e}(\mathrm{~dB})$ & $\xi^{\prime}$ & $\xi$ & $K_{1}$ & $K_{0}$ & $M$ \\
\hline \hline RZ with EF & -18.0 & 3.49 & 0.44 & 3.51 & 3.51 & 38.8 \\
\hline RZ w/o EF & -18.0 & 5.91 & 0.74 & 3.17 & 3.17 & 17.7 \\
\hline NRZ with EF & -11.3 & 1.89 & 0.24 & 2.88 & 2.68 & 38.8 \\
\hline NRZ w/o EF & -11.9 & 1.95 & 0.25 & 2.81 & 2.79 & 17.7 \\
\hline
\end{tabular}

provide an important baseline for future studies that will include pattern dependences. The RZ pulse was Gaussian-shaped with a FWHM of 23 ps, and the NRZ pulses had a rise time of 34 ps. The optical extinction ratio was $18 \mathrm{~dB}$ for the RZ signal and 12 $\mathrm{dB}$ for the NRZ signal. At the receiver, an optical preamplifier increased the signal and noise power so that the optical noise dominated the electrical noise. The total power into the $20-\mathrm{GHz}$ photodetector was kept fixed at $-2 \mathrm{dBm}$ by tuning an attenuator. The FWHM of the Gaussian optical filter was $187 \mathrm{GHz}$, and either a fifth-order low-pass electrical Bessel filter with a 3$\mathrm{dB}$ bandwidth of $7 \mathrm{GHz}$ or no electrical filter was used in the receiver. The photodetector and the scope limited the bandwidth of the electric signal when no electrical filter was used. We experimentally verified that the combined frequency response of the photodetector and the scope was well approximated by a Gaussian filter with a bandwidth of $15 \mathrm{GHz}$. The noise-equivalent bandwidth of the OSA was $25 \mathrm{GHz}$. A high-speed sampling oscilloscope was used to measure the $Q$ factor at the same time that the OSNR was measured. In Fig. 3, the curves show results obtained using (50), and the symbols show the experimental results. The dotted-dashed curve and the diamonds show the results for an RZ format with the electrical filter. The solid curve and circles show the results for the RZ format without the electrical filter. The dashed curve and squares show the results for the NRZ format with the electrical filter, and the dotted curve and triangles show the results for the NRZ format without the electrical filter. The parameters in (50) for the modulation formats shown in Fig. 3 are described in Table I.

In Fig. 3, we observe that the performance of the RZ format is less sensitive than is the performance of the NRZ format to variations in the characteristics of the receiver. Since the noise is unpolarized, $\Gamma_{\mathrm{ASE}-\mathrm{ASE}}=1$, and $\Gamma_{\mathrm{S}-\mathrm{ASE}}=1 / 2$. The results that we obtain using the formula (50) are in good agreement with the experimental results shown in this figure. An increase of the bandwidth of the electrical filter increases the amount of noise in the decision circuit which degrades the system performance. On the other hand, for systems with a 10-Gb/s RZ format, increasing the electrical bandwidth from 7 to $15 \mathrm{GHz}$ also reduces the broadening of the RZ pulses, and thereby increases the electric current due to the signal in the marks. However, this same effect does not occur in systems that use the NRZ format, since the NRZ pulses have a much narrower bandwidth.

In the second set of experiments, we investigated the effect of partially polarized noise on the system performance. In Fig. 4, we plot the maximum and the minimum values of the $Q$ factor as a function of the degree of polarization of the noise at the receiver $\mathrm{DOP}_{n}$. These simulation and experimental results are for 


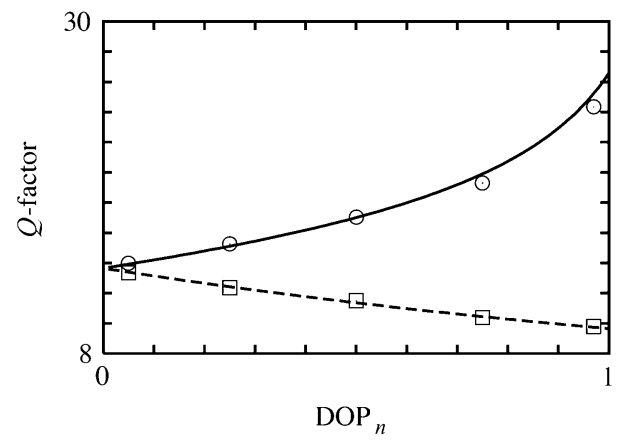

Fig. 4. Comparison of the $Q$ factor as a function of the degree of polarization of the noise $\mathrm{DOP}_{n}$ obtained using (47) with experiments for different polarization states of the signal and of the noise. The solid curve shows the result obtained using (47), and the circles show the experimental results when the Jones vectors of the signal and the polarized part of the noise are orthogonal. The dashed curve shows the result obtained using (47), and the squares show the experimental results when the signal is co-polarized with the polarized part of the noise.

an RZ signal with optical noise added by two EDFAs without input power [31]. The RZ pulse was Gaussian-shaped with a FWHM of 23 ps. In the simulations, we used a perfect optical extinction ratio. The receiver consisted of a photodetector, an optical filter, and an electrical amplifier. We used the method described in [5] to obtain the $Q$ factor from the BER margin measurements. Bergano et al. [5] showed that the $Q$ factor obtained from BER margin measurements is well correlated to the BER. The first optical amplifier generated unpolarized noise, and the noise generated by the second optical amplifier was polarized by passing it through a polarizer and a polarization controller. The degree of polarization of the noise $\mathrm{DOP}_{n}$ was controlled by adjusting two variable attenuators that follow the two optical amplifiers. The direction on the Poincaré sphere of the normalized Stokes' vector $\mathbf{s}_{n}^{(p)}$ of the polarized part of the noise was adjusted by the polarization controller that follows the polarizer. The SNR of the electric current of the marks $\mathrm{SNR}_{1}$, which was defined in (36), was fixed at $10.9 \mathrm{~dB}$. The FWHM of the Gaussian optical filter was $187 \mathrm{GHz}$, and the frequency response of the photodetector and electrical amplifier was modeled by a Gaussian electrical filter with a 3-dB bandwidth of $15 \mathrm{GHz}$.

In the experiment, for each value of $\mathrm{DOP}_{n}$, we varied the angle between the Stokes' vectors of the signal and the polarized part of the noise and recorded the maximum value $Q_{\max }$ and minimum value $Q_{\min }$ of the $Q$ factor. In the simulations, we obtained $Q_{\max }$ by choosing the Stokes' vectors of the signal and the polarized part of the noise to be antiparallel $\mathbf{s}_{s} \cdot \mathbf{s}_{n}^{(p)}=$ -1 so that the corresponding Jones vectors are orthogonal to each other. Similarly, we obtained $Q_{\min }$ by choosing the Stokes' vectors $\mathbf{s}_{s}$ and $\mathbf{s}_{n}^{(p)}$ to be parallel $\mathbf{s}_{s} \cdot \mathbf{s}_{n}^{(p)}=+1$.

In Fig. 4, the curves show results obtained using (47), and the symbols show the experimental results. We observed an excellent agreement between the formula (47) and the experimental results. The solid curve and circles show the maximum value of the $Q$ factor versus $\mathrm{DOP}_{n}$, while the dashed curve and squares show the minimum value of the $Q$ factor. As the DOP of the noise increases from 0 to 1 , we observe a dramatic increase in the range $\left[Q_{\min }, Q_{\max }\right]$ of the $Q$ factor. For this receiver, the parameters in (47) are $\mu=38.6, \kappa_{1}=1.7, \kappa_{0}=0$, and $\alpha_{e}=0$. When the noise is depolarized $\left(\mathrm{DOP}_{n}=0\right)$, one has $\Gamma_{\mathrm{ASE}-\mathrm{ASE}}=1$, and $\Gamma_{\mathrm{S}-\mathrm{ASE}}=1 / 2$. When the noise is completely polarized $\left(\mathrm{DOP}_{n}=1\right)$, one has $\Gamma_{\mathrm{ASE}-\mathrm{ASE}}=1 / 2$ so that $\Gamma_{\mathrm{S}-\mathrm{ASE}}=1$ when the noise is co-polarized with the signal, and $\Gamma_{\mathrm{S}-\mathrm{ASE}}=0$ when the polarization state of the noise is orthogonal to the signal. The results that we show in Figs. 2 and 4 illustrate the significant impact that partially polarized noise can have on the performance of an optical fiber transmission system. Typical values for the PDL per optical amplifier in optical fiber systems range from 0.1 to $0.2 \mathrm{~dB}$ [2], which can partially polarize the optical noise in the transmission line. In a prototypical system described in [15], which has 270 amplifiers with $0.15 \mathrm{~dB}$ of PDL per amplifier, the average-noise DOP at the receiver is equal to 0.15 . However, in this prototypical system, the probability that the DOP of the noise exceeds 0.3 is larger than $10^{-3}$.

\section{CONCLUSION}

In conclusion, a formula has been derived that relates the $Q$ factor to the OSNR for amplitude-shift-keyed optical fiber transmission systems with arbitrary optical pulse shapes, arbitrary receiver characteristics, and arbitrarily polarized noise using a realistic receiver model. In this paper, the enhancement factor and three other parameters were also defined that explicitly quantify the performance of different modulation formats. This method was validated by comparison with back-to-back $10-\mathrm{Gb} / \mathrm{s}$ experiments and Monte Carlo simulations. The proposed method was used to show that the RZ format is less sensitive to variations in the receiver than the NRZ format, and this conclusion was experimentally validated. The method developed can be used in receiver optimization studies, and it can also be applied to the study of other amplitude-shift-keyed formats. This method accounts for partially polarized noise, which can be produced by PDL and PDG in optical amplifiers. Computer simulations and laboratory experiments were used to quantify the strong dependence of the system performance on the polarization state of the noise relative to that of the signal when the optical noise is partially polarized. Therefore, this method can be used in combination with reduced system models to study the combined effect that the polarization effects have on the system performance. Since the method is computationally efficient, it can also be used as a baseline for the analysis of transmission systems that have pattern dependences due to effects such as chromatic dispersion, PMD, and the Kerr nonlinearity.

\section{APPENDIX}

In this Appendix, tables are provided for the effective number of noise modes $\mu$; the signal-noise beating parameters; $\kappa_{0}$ and $\kappa_{1}$ for the spaces and marks, respectively; and the enhancement factor $\xi$. The results are for back-to-back systems with typical RZ and NRZ modulation formats and typical receiver filters. These tables allow one to easily calculate the $Q$ factor for such systems without having to compute the multiple integrals in (26) and (29).

The parameters $\mu, \kappa_{0}, \kappa_{1}$ and $\xi$ satisfy the following scaling laws. First, they are independent of the power in the signal. 
TABLE II

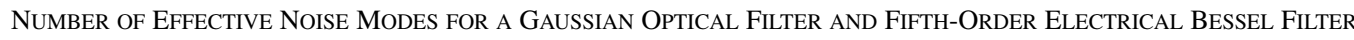

\begin{tabular}{l||l|l|l|l|l|l|l|l}
\hline$\Delta \omega_{e} / \Delta \omega_{0}$ & 0.08 & 0.10 & 0.12 & 0.16 & 0.20 & 0.30 & 0.40 & 0.80 \\
\hline$\mu$ & 18.22 & 14.62 & 12.23 & 9.26 & 7.50 & 5.21 & 4.11 & 2.6 \\
\hline
\end{tabular}

TABLE III

COMmon Value of the Signal-Noise BeAting PARAMETERs $\kappa_{0}$ AND $\kappa_{1}$ FOR THE RZ MOdUlation Format With a Gaussian Optical Filter and Fifth-Order Electrical Bessel Filter. First and SECOND SUBSCRIPTS ARE $\Delta \omega_{o, \min }$ FOR $\kappa_{0}$ AND $\kappa_{1}$, RESPECTIVELY

\begin{tabular}{l||l|l|l|l|l|l|l|l}
\hline \multicolumn{1}{l||}{$\tau_{s} \Delta \omega_{o}$} & \multicolumn{7}{c}{$\Delta \omega_{e} / \Delta \omega_{o}$} \\
\hline & 0.08 & 0.10 & 0.12 & 0.16 & 0.20 & 0.30 & 0.40 & 0.80 \\
\hline \hline 0.5 & $2.73_{60,40}$ & $2.71_{50,40}$ & $2.68_{40,30}$ & $2.63_{30,10}$ & $2.56_{30,10}$ & $2.36_{20,10}$ & $2.18_{20,10}$ & $1.73_{10,10}$ \\
\hline 1.0 & $3.18_{60,40}$ & $3.13_{50,40}$ & $3.07_{40,30}$ & $2.93_{30,10}$ & $2.79_{30,10}$ & $2.47_{10,10}$ & $2.22_{10,10}$ & $1.73_{10,10}$ \\
\hline 1.5 & $3.32_{60,40}$ & $3.23_{50,10}$ & $3.14_{50,10}$ & $2.95_{40,10}$ & $2.78_{30,10}$ & $2.44_{10,10}$ & $2.21_{10,10}$ & $1.73_{10,10}$ \\
\hline 2.0 & $3.33_{60,40}$ & $3.21_{50,10}$ & $3.09_{50,10}$ & $2.89_{40,10}$ & $2.73_{40,10}$ & $2.42_{10,10}$ & $2.20_{10,10}$ & $1.73_{10,10}$ \\
\hline 2.5 & $3.28_{70,40}$ & $3.15_{60,10}$ & $3.03_{50,10}$ & $2.84_{50,10}$ & $2.69_{40,10}$ & $2.41_{10,10}$ & $2.19_{10,10}$ & $1.73_{10,10}$ \\
\hline 3.0 & $3.22_{70,40}$ & $3.08_{60,10}$ & $2.97_{60,10}$ & $2.79_{50,10}$ & $2.66_{10,10}$ & $2.40_{10,10}$ & $2.19_{10,10}$ & $1.73_{10,10}$ \\
\hline
\end{tabular}

TABLE IV

ENHANCEMENT FACTOR $\xi$ FOR THE RZ MOdULATION FoRMat, With a GAUSSIAN OPTICAL Filter AND FifTH-ORDER EleCtRICAL BeSSEL Filter. SUbSCRIPTS SHOW tHE CORRESPONDING VALUES OF $\Delta \omega_{o, \text { min }}$

\begin{tabular}{l||l|l|l|l|l|l|l|l}
\hline \multicolumn{1}{l||}{$\tau_{s} \Delta \omega_{o}$} & \multicolumn{7}{c}{$\Delta \omega_{e} / \Delta \omega_{o}$} \\
\hline & 0.08 & 0.10 & 0.12 & 0.16 & 0.20 & 0.30 & 0.40 & 0.80 \\
\hline \hline 0.5 & $0.75_{50}$ & $0.93_{40}$ & $1.11_{30}$ & $1.46_{30}$ & $1.79_{10}$ & $2.50_{10}$ & $3.05_{10}$ & $4.15_{10}$ \\
\hline 1.0 & $0.90_{50}$ & $1.11_{40}$ & $1.31_{30}$ & $1.67_{30}$ & $1.99_{20}$ & $2.56_{20}$ & $2.90_{20}$ & $3.38_{20}$ \\
\hline 1.5 & $0.92_{50}$ & $1.12_{40}$ & $1.30_{30}$ & $1.60_{30}$ & $1.83_{30}$ & $2.18_{30}$ & $2.35_{30}$ & $2.55_{30}$ \\
\hline 2.0 & $0.90_{50}$ & $1.08_{40}$ & $1.23_{40}$ & $1.46_{40}$ & $1.61_{40}$ & $1.82_{40}$ & $1.91_{40}$ & $2.01_{40}$ \\
\hline 2.5 & $0.87_{50}$ & $1.02_{50}$ & $1.14_{50}$ & $1.31_{50}$ & $1.41_{50}$ & $1.54_{50}$ & $1.59_{50}$ & $1.64_{50}$ \\
\hline 3.0 & $0.83_{60}$ & $0.96_{60}$ & $1.05_{60}$ & $1.17_{60}$ & $1.24_{60}$ & $1.32_{60}$ & $1.35_{60}$ & $1.38_{60}$ \\
\hline
\end{tabular}

TABLE V

VAlues $\left(\kappa_{0}, \kappa_{1}\right)$ OF the Signal-Noise Beating Parameters For the NRZ Modulation Format With a 50-GHz Gaussian Optical Filter and FifTH-Order EleCtrical BesSel Filter

\begin{tabular}{l||l|l|l|l|l|l}
\hline \multicolumn{1}{l||}{ Rise Time(ps) } & \multicolumn{7}{c}{$\Delta \omega_{e} / \Delta \omega_{o}$} \\
\hline & 0.08 & 0.12 & 0.16 & 0.20 & 0.30 & 0.40 \\
\hline \hline 20 & $(1.38,3.08)$ & $(2.19,2.80)$ & $(2.63,2.66)$ & $(2.58,2.58)$ & $(2.34,2.37)$ & $(2.18,2.18)$ \\
\hline 30 & $(1.49,3.09)$ & $(2.14,2.82)$ & $(2.58,2.68)$ & $(2.56,2.58)$ & $(2.35,2.37)$ & $(2.18,2.18)$ \\
\hline 40 & $(1.57,3.10)$ & $(2.12,2.84)$ & $(2.57,2.70)$ & $(2.57,2.59)$ & $(2.35,2.37)$ & $(2.17,2.18)$ \\
\hline 50 & $(1.69,3.10)$ & $(2.14,2.86)$ & $(2.44,2.71)$ & $(2.48,2.61)$ & $(2.33,2.38)$ & $(2.17,2.18)$ \\
\hline 60 & $(1.81,3.09)$ & $(2.19,2.86)$ & $(2.40,2.72)$ & $(2.44,2.61)$ & $(2.33,2.38)$ & $(2.16,2.18)$ \\
\hline
\end{tabular}

Second, for a given pulse shape and given optical and electrical filter shapes

$$
\mu\left(\Delta \omega_{e}, \Delta \omega_{o}\right)=\mu\left(\Delta \omega_{e} / \Delta \omega_{o}\right)
$$

that is, $\mu$ only depends on $\Delta \omega_{e} / \Delta \omega_{o}$ and not on $\left(\Delta \omega_{e}, \Delta \omega_{o}\right)$, and

$$
\begin{aligned}
\kappa_{i}\left(\Delta \omega_{e}, \Delta \omega_{o}, \tau_{s}\right) & =\kappa_{i}\left(\Delta \omega_{e} / \Delta \omega_{o}, \tau_{s} \Delta \omega_{o}\right), \quad i=1,2 \\
\xi\left(\Delta \omega_{e}, \Delta \omega_{o}, \tau_{s}\right) & =\xi\left(\Delta \omega_{e} / \Delta \omega_{o}, \tau_{s} \Delta \omega_{o}\right)
\end{aligned}
$$

where $\Delta \omega_{e}$ is the $f_{3 \mathrm{~dB}}$ bandwidth of the electrical filter, $\Delta \omega_{o}$ is the full-width at half-maximum (FWHM) of the optical filter, and $\tau_{s}$ is the FWHM of the optical pulse. In the derivation of (59), it was assumed that there are no bit-patterning effects, i.e., (59) holds for an isolated mark or space.

For all the results presented here, a Gaussian optical filter and a fifth-order electrical Bessel filter were used. In Table II, values are provided for $\mu$ as a function of $\Delta \omega_{e} / \Delta \omega_{0}$. Table III shows the common value of $\kappa_{0} \simeq \kappa_{1}$ as a function of $\Delta \omega_{e} / \Delta \omega_{o}$ and $\tau_{s} \Delta \omega_{o}$ for a $10-\mathrm{Gb} / \mathrm{s}$ RZ modulation format with Gaussianshaped pulses and an optical extinction ratio of $15 \mathrm{~dB}$. Due to bit-patterning effects, the values in Table III are only valid for

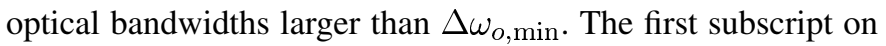
each of the values in Table III shows the values of $\Delta \omega_{o \text {,min }}$ for which the relative error between the actual values of $\kappa_{0}$ at all bandwidths $\Delta \omega_{o} \geq \Delta \omega_{o, \min }$ and the value in the table are less than $10 \%$. The second subscript shows the corresponding value of $\Delta \omega_{o, \min }$ for $\kappa_{1}$. In most cases, the relative error is less than about $2 \%$ when $\Delta \omega_{o} \geq \Delta \omega_{o, \min }+15 \mathrm{GHz}$. Table IV shows the corresponding values of the enhancement factor $\xi$, with the subscript indicating the value of $\Delta \omega_{o, \min }$. For all the results in this Appendix, a De Bruijn pseudorandom bit pattern of length $2^{6}$ was used, the time and frequency domains were discretized using 8192 points, and the parameters were computed using the space with the largest voltage and the mark with the smallest voltage in the electrically filtered signal at the clock-recovery time. 
TABLE VI

ENHANCEMENT FACTOR $\xi$ FOR THE NRZ MODULATION FORMAT, With A 50-GHz GAUSSIAN OPTICAL FILTER AND FIFTH-ORDER ELECTRICAL BESSEL FILTER

\begin{tabular}{l||l|l|l|l|l|l}
\hline \multicolumn{1}{l||}{ Rise Time } & \multicolumn{7}{c}{$\Delta \omega_{e} / \Delta \omega_{o}$} \\
\hline & 0.08 & 0.12 & 0.16 & 0.20 & 0.30 & 0.40 \\
\hline \hline 20 & 0.77 & 0.90 & 0.93 & 0.94 & 0.94 & 0.94 \\
\hline 30 & 0.76 & 0.89 & 0.94 & 0.95 & 0.95 & 0.95 \\
\hline 40 & 0.74 & 0.87 & 0.92 & 0.94 & 0.96 & 0.96 \\
\hline 50 & 0.72 & 0.85 & 0.90 & 0.93 & 0.95 & 0.95 \\
\hline 60 & 0.69 & 0.81 & 0.87 & 0.90 & 0.92 & 0.93 \\
\hline
\end{tabular}

Table $\mathrm{V}$ shows the values of $\left(\kappa_{0}, \kappa_{1}\right)$ for a $10-\mathrm{Gb} / \mathrm{s}$ NRZ modulation format with an optical extinction ratio of $15 \mathrm{~dB}$ and an optical filter bandwidth of $\Delta \omega_{o}=50 \mathrm{GHz}$. The different rows in the table correspond to different rise times for the NRZ signal. In Table VI, the corresponding values of $\xi$ are shown. It does not make sense to scale the widths of the pulses of the NRZ signal, unless one also scales the bit rate. To investigate how the parameter values depend on the bandwidth of the optical filter, simulations were also performed for the NRZ format using an optical filter with an FWHM of $25 \mathrm{GHz}$. It was found that the values $\kappa_{0}^{25}, \kappa_{1}^{25}$ and $\xi^{25}$ for the $25-\mathrm{GHz}$ bandwidth optical filter are related to the entries $\kappa_{0}^{50}, \kappa_{1}^{50}$ and $\xi^{50}$ in Tables V and VI by

$$
\begin{aligned}
& \kappa_{0}^{25}=0.89 \kappa_{0}^{50} \pm 0.13 \\
& \kappa_{1}^{25}=0.87 \kappa_{0}^{50} \pm 0.07 \\
& \xi^{25}=1.96 \xi^{50} \pm 0.04 .
\end{aligned}
$$

\section{REFERENCES}

[1] D. Marcuse, "Derivation of analytical expressions for the bit-error probability in lightwave systems with optical amplifiers," J. Lightw. Technol., vol. 8, no. 12, pp. 1816-1823, Dec. 1990.

[2] I. P. Kaminow and I. L. Koch, Optical Fiber Telecommunications. San Diego, CA: Academic, 1997, vol. III-B.

[3] I. P. Kaminow and T. Li, Optical Fiber Telecommunications. San Diego, CA: Academic, 2002, vol. IV-B.

[4] P. A. Humblet and M. Azizog̃lu, "On the bit error rate of lightwave systems with optical amplifiers," J. Lightw. Technol., vol. 9, no. 11, pp. 1576-1582, Nov. 1991

[5] N. S. Bergano, F. W. Kerfoot, and C. R. Davidson, "Margin measurements in optical amplifier systems," IEEE Photon. Technol. Lett., vol. 5, no. 3, pp. 304-306, Mar. 1993.

[6] E. Golovchenko, "The challenges of designing long-haul WDM systems," presented at the Tutorial Sessions Optical Fiber Communication Conf. (OFC 2002), 2002, Paper TuL.

[7] P. J. Winzer, M. Pfennigbauer, M. M. Strasser, and W. R. Leeb, "Optimum filter bandwidth for optically preamplified NRZ receivers," $J$. Lightw. Technol., vol. 19, no. 9, pp. 1263-1273, Sep. 2001.

[8] I. T. Lima Jr., A. O. Lima, J. Zweck, and C. R. Menyuk, "Performance characterization of chirped return-to-zero modulation format using an accurate receiver model," IEEE Photon. Technol. Lett., vol. 15, no. 4, pp. 608-610, Apr. 2003.

[9] J. L. Rebola and A. V. T. Cartaxo, "Power penalty assessment in optically preamplified receivers with arbitrary optical filtering and signal-dependent noise dominance," J. Lightw. Technol., vol. 20, no. 3, pp. 401-408, Mar. 2002.

[10] — " $Q$-factor estimation and impact of spontaneous-spontaneous beat noise on the performance of optically preamplified systems with arbitrary optical filtering," J. Lightw. Technol., vol. 21, no. 1, pp. 87-95, Jan. 2003.

[11] E. Lichtman, "Limitations imposed by polarization-dependent gain and loss on all-optical ultralong communication systems," J. Lightw. Technol., vol. 13, no. 5, pp. 906-913, May 1995.

[12] A. Mecozzi and M. Shtaif, "The statistics of polarization-dependent loss in optical communication systems," IEEE Photon. Technol. Lett., vol. 14, no. 3, pp. 313-315, Mar. 2002.
[13] P. Lu, S. Mihailov, L. Chen, and X. Bao, "Importance sampling for the combination of polarization mode dispersion and polarization dependent loss," in Proc. Optical Fiber Communication Conf. (OFC 2003), vol. 1, 2003, pp. 5-6. Paper MF5.

[14] B. Huttner, C. Geiser, and N. Gisin, "Polarization-induced distortions in optical fiber networks with polarization-mode dispersion and polarization-dependent losses," IEEE J. Select. Topics Quantum Electron., vol. 6, no. 2, pp. 317-329, Mar-Apr. 2000.

[15] D. Wang and C. R. Menyuk, "Calculation of penalties due to polarization effects in a long-haul WDM system using a stokes parameter model," $J$. Lightw. Technol., vol. 19, no. 4, pp. 487-494, Apr. 2001.

[16] I. T. Lima Jr., "Investigation of the performance degradation due to polarization effects in optical fiber communications systems," Ph.D. dissertation, Dept. Comp. Sci. Elect. Eng., Univ. Maryland Baltimore County, Baltimore, MD, Dec. 2003.

[17] Y. Sun, A. O. Lima, I. T. Lima Jr., J. Zweck, L. Yan, C. R. Menyuk, and G. M. Carter, "Statistics of the system performance in scrambled recirculating loop with PDL and PDG," IEEE Photon. Technol. Lett., vol. 15, no. 8, pp. 1067-1069, Aug. 2003.

[18] I. T. Lima Jr., A. O. Lima, J. Zweck, and C. R. Menyuk, "Efficient computation of outage probabilities due to polarization effects in a WDM system using a reduced stokes model and importance sampling," IEEE Photon. Technol. Lett., vol. 15, no. 1, pp. 45-47, Jan. 2003.

[19] C. J. Anderson and J. A. Lyle, "Technique for evaluating system performance using $Q$ in numerical simulations exhibiting intersymbol interference," Electron. Lett., vol. 30, no. 1, pp. 71-72, 1994.

[20] R. Holzlöhner, C. R. Menyuk, V. S. Grigoryan, and W. L. Kath, "Accurate calculation of eye diagrams and error rates in long-haul transmission systems using linearization," J. Lightw. Technol., vol. 20, no. 3, pp. 389-400, Mar. 2002.

[21] R. Holzlöhner, C. R. Menyuk, W. L. Kath, and V. S. Grigoryan, "Efficient and accurate calculation of eye diagrams and bit-error rates in a single-channel CRZ system," IEEE Photon. Technol. Lett., vol. 14, no. 8, pp. 1079-1081, Aug. 2002.

[22] L. Kazovsky, S. Benedetto, and A. Willner, Optical Fiber Communication Systems. Norwood, MA: Artech House, 1996.

[23] E. Desurvire, Erbium Doped Fiber Amplifiers. New York, NY: Wiley, 1994.

[24] B. E. A. Saleh and M. C. Teich, Fundamentals of Photonics. New York: Wiley, 1991

[25] M. Born and E. Wolf, Principles of Optics, Cambridge, U.K.: Cambridge Univ. Press, 1999.

[26] P. R. Trischitta and E. L. Varma, Jitter in Digital Transmission Systems. Boston, MA: Artech House, 1989.

[27] S. Norimatsu and M. Maruoka, "Accurate $Q$-factor estimation of optically amplified systems in the presence of waveform distortions," $J$. Lightw. Technol., vol. 20, no. 1, pp. 19-27, Jan. 2002.

[28] I. P. Kaminow and I. L. Koch, Optical Fiber Telecommunications. San Diego, CA: Academic, 1997, vol. III-A.

[29] R. E. Walpole and R. H. Myers, Probability and Statistics for Engineers and Scientists. New York, NY: Macmillan, 1993.

[30] H. Jiao, I. T. Lima Jr., A. O. Lima, Y. Sun, J. Zweck, L. Yan, C. R. Menyuk, and G. M. Carter, "Experimental validation of an accurate receiver model for systems with unpolarized noise," in Proc. Conf. Lasers and Electro-Optics (CLEO), Baltimore, MD, Jun. 1-6, 2003, paper CThJ1.

[31] Y. Sun, I. T. Lima Jr., A. O. Lima, H. Jiao, J. Zweck, L. Yan, C. R. Menyuk, and G. M. Carter, "System performance variations due to partially polarized noise in a receiver," IEEE Photon. Technol. Lett., vol. 15, no. 11, pp. 1648-1650, Nov. 2003. 
Ivan T. Lima, Jr. (M'04) received the B.Sc. degree in electrical engineering from the Federal University of Bahia (UFBA), Salvador, Brazil, in 1995, the M.Sc. degree in electrical engineering from the State University of Campinas (UNICAMP), Campinas, Brazil, in 1998, and the Ph.D. degree in electrical engineering in the field of photonics from the University of Maryland, Baltimore County, in 2003.

From 1986 to 1996, he was with Banco do Brasil (Bank of Brazil), where he served as the Information Technology Advisor of the State Superintendence of Bahia, Brazil. He was a Research Assistant in the Optical Fiber Communications Laboratory at the University of Maryland Baltimore County from 1998 to 2003. In 2003, he became an Assistant Professor in the Department of Electrical and Computer Engineering at North Dakota State University, Fargo. His research interests have been devoted to the modeling of transmission effects and receivers in optical fiber communications systems. He has authored or coauthored 20 archival journal papers, 38 conference contributions, one book chapter, and one U.S. patent.

Dr. Lima received the 2003 IEEE LEOS Graduate Student Fellowship Award, and he was a corecipient of the Venice Summer School on Polarization Mode Dispersion Award. In 2004, he was co-instructor of the Short Course SC210: Hands-On Polarization Measurement Workshop, which was offered at the Optical Fiber Communications Conference and Exposition (OFC) 2004, Los Angeles, CA. He is a Member of the IEEE Lasers and Electro-Optics Society (LEOS) and of the Optical Society of America.

Aurenice O. Lima ( $\left.\mathrm{S}^{\prime} 00\right)$ received the B.Sc. degree in electrical engineering from the Federal University of Bahia (UFBA), Salvador, Brazil, in 1995, and the M.Sc. degree in electrical engineering from the State University of Campinas (Unicamp), Campinas, Brazil, in 1998. She is currently working toward the $\mathrm{Ph} . \mathrm{D}$. degree in electrical engineering in the Department of Computer Science and Electrical Engineering, the University of Maryland, Baltimore County.

In 2000, she became a Research Assistant in the Optical Fiber Communications Laboratory at the University of Maryland, Baltimore County. In 2005, she also became an Adjunct Faculty Member in the Department of Electrical and Computer Engineering, North Dakota State University, Fargo. Her current research interests include modeling and statistical analysis of polarization effects, and signal processing for optical fiber communication systems. She is currently focusing her research on the study of advanced Monte Carlo methods for computation of penalties induced by polarization mode dispersion in optical fiber transmission systems. She has authored or co-authored 10 archival journal publications and twenty-one conference contributions.

Ms. Lima received the Venice Summer School on Polarization Mode Dispersion Award in 2003. In 1996 and 1998, she was awarded with Graduate Scholarships from the Brazilian Ministry of Education and from the Brazilian Ministry of Science and Technology, respectively. Ms. Lima is a Student Member of the IEEE Lasers and Electro-Optics Society (LEOS) and of the IEEE Women in Engineering Society.

Yu Sun, photograph and biography not available at the time of publication.
Hua Jiao, photograph and biography not available at the time of publication.

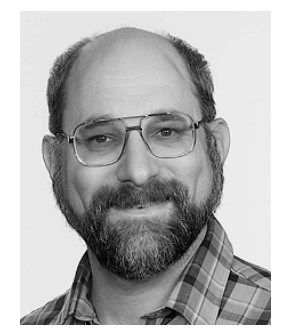

Curtis R. Menyuk (S'88-F'98) was born on March 26, 1954. He received the B.S. and M.S. degrees from the Massachusetts Institute of Technology, Cambridge, in 1976, and the Ph.D. degree from the University of California, Los Angeles, in 1981.

$\mathrm{He}$ has worked as a Research Associate at the University of Maryland, College Park, and at Science Applications International Corporation, McLean, VA. In 1986, he became an Associate Professor in the Department of Electrical Engineering at the University of Maryland Baltimore County (UMBC), and he was the Founding Member of this department. In 1993, he was promoted to Professor. He was on partial leave from UMBC from fall 1996 until fall 2002. From 1996 to 2001, he worked part-time for the Department of Defense, co-directing the Optical Networking program at the DoD Laboratory for Telecommunications Sciences, Adelphi, MD, from 1999 to 2001. In 2001-2002, he was Chief Scientist at PhotonEx Corporation. For the last 17 years, his primary research area has been theoretical and computational studies of fiber optic communications. He has authored or coauthored more than 180 archival journal publications as well as numerous other publications and presentations. He has also edited two books. The equations and algorithms that he and his research group at UMBC have developed to model optical fiber transmission systems are used extensively in the telecommunications industry.

Dr. Menyuk is a Member of the Society for Industrial and Applied Mathematics and the American Physical Society. He is a Fellow of the Optical Society of America (OSA), and is a former UMBC Presidential Research Professor.

Gary M. Carter, photograph and biography not available at the time of publication. 